\title{
Harmonic and pulsed eddy current testing methods as tools for surface and subsurface defect evaluation
}

\author{
Lukáš Behúñ ${ }^{*}$, Karel Slobodník ${ }^{* *}$
}

\begin{abstract}
In most publications eddy current testing (ECT) methods are said to be suitable for surface defects up to several millimeters. This paper aims to evaluate the detection capabilities of eddy current testing based on two examples of modern approach. One of them is a harmonic eddy current method with use of wavelet transformation and neural network. The second method is pulsed eddy current testing (PECT) with use of evaluation script based on cross-correlation of measured data with the set of known reference signals. Two samples with five artificial defects each were investigated from the near and far side. Geometric parameters of every defect were then estimated. Results show that both methods are limited by the skin effect. However, the PECT with use of cross-correlation exhibits detection capabilities of subsurface defects in significant depth. On the other hand, harmonic eddy current testing shows lower errors in dimensions estimation for set of surface defects.
\end{abstract}

K e y w ord s: ECT, PECT, eddy current, cross-correlation, neural network, wavelet transform

\section{Introduction}

Modern industry applications often require detection of structural defects in objects without compromising the structural integrity of the system. This type of evaluation is called nondestructive testing and include wide variety of methods based on use of different physical fields. Nondestructive testing is mostly used during a continuous examination in production line, as part of periodic inspections or when cost of destructive testing would be too high. Eddy current testing (ECT) is one of most widely used methods in non-destructive testing, but its use is limited to conductive materials. ECT is based on the elec- tromagnetic induction phenomenon. When time-varying electric current is applied to a coil, a non-stationary electromagnetic field is developed in its proximity. If electrically conductive body is placed in the range of nonstationary electromagnetic field, eddy currents are generated in the body as a product of electromotive force. Flow of eddy currents produce secondary electromagnetic field, which has the opposite direction in comparison to primary electromagnetic field of the coil. If the defect is presented in the structure of tested sample, eddy current flow is altered. Alteration in eddy current flow leads to changes in secondary electromagnetic field. By measuring

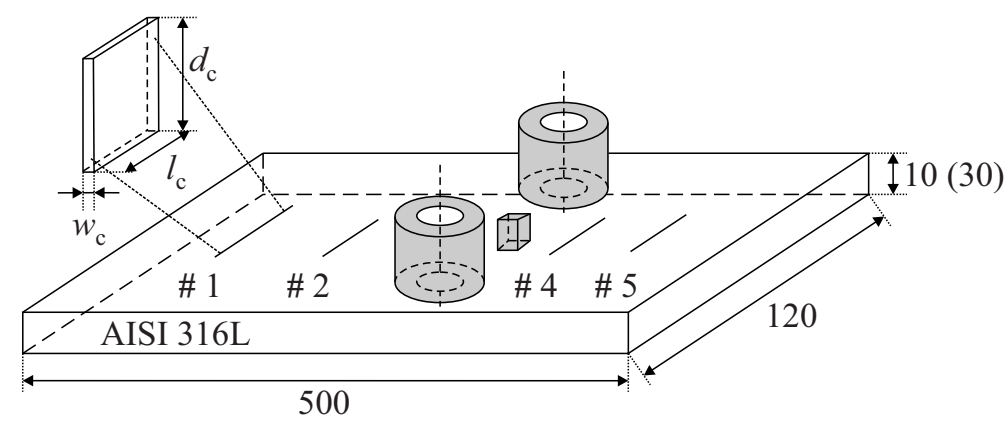

Fig. 1. Experimental configuration of the investigated steel specimen

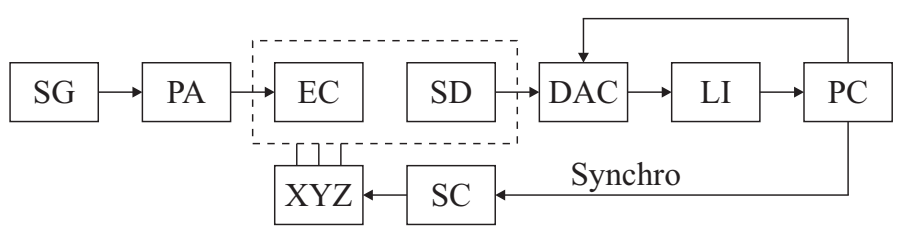

Fig. 2. Block diagram of the experimental setup: SG - signal generator, PA - power amplifier, EC - excitation coils, SD - sensing device, DAC - data acquisition card, XYZ - 3-axes stage, LI - lock-in amplifier, SC - stage controller, PC - personal computer

* Department of Electromagnetic and Biomedical Engineering, University of Žilina, Žilina, Slovakia, ** Department of Electrical Engineering Theory, University of West Bohemia, Pilsen, Czech Republic, karelsl@kte.zcu.cz,karelsl@students.zcu.cz 


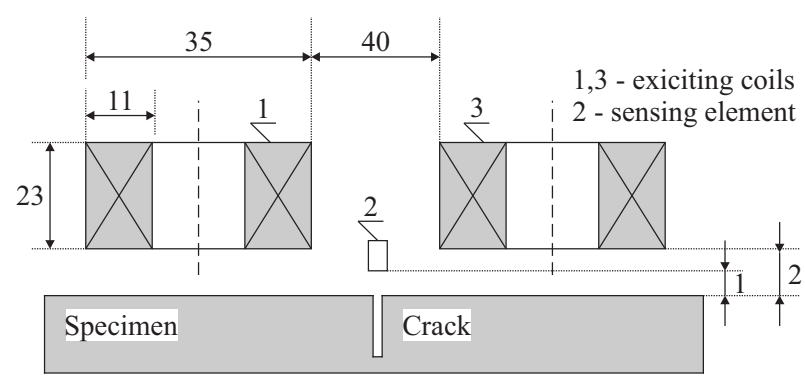

Fig. 3. Configuration of the ECT probe

the intensity of electromagnetic field, information about the material defect is obtained $[1,4]$.

In majority of industry applications harmonic excitation of primary magnetic field is used, although in last few years pulsed eddy current testing (PECT) start to be applied. The pulsed excitation signal is easy to generate, efficient due to low power loss in switching mode and contains wide range of frequencies. PECT is currently mostly used in surface cracks and corrosion detection under insulation layer and wall thickness measurement $[5,6]$.

Table 1. Investigated rectangular-shape defects according Fig. 1, all dimensions are in $\mathrm{mm}$

\begin{tabular}{cccccc}
\hline \multirow{2}{*}{ Defect } & \multicolumn{2}{c}{ length $=30$} & & \multicolumn{2}{c}{ length $=10$} \\
\cline { 2 - 3 } \cline { 5 - 6 } & width & depth & & width & depth \\
\hline 1 & 0.5 & 5 & & 0.55 & 1 \\
2 & 0.55 & 10.3 & & 0.55 & 3 \\
3 & 0.6 & 16.3 & & 0.6 & 5 \\
4 & 0.7 & 20.5 & & 0.6 & 7 \\
5 & 0.7 & 24.5 & & 0.6 & 9 \\
\hline
\end{tabular}

length $=$ thickness

\section{Experimental set-up}

Conductive stainless steel (AISI316L) plates specimen are inspected in this study with thickness $h=10 \mathrm{~mm}$ and $h=30 \mathrm{~mm}$, and having the conductivity of $\sigma=$ $1.4 \mathrm{MS} / \mathrm{m}$ and the relative permeability of $\mu_{\mathrm{r}}=1$. Each specimen contains five rectangular shaped defects of zero conductivity, Fig. 1. The specimens have the defects with following geometry, see Tab. 1 .

Surface area of $s=60 \mathrm{~mm} \times 60 \mathrm{~mm}$ in the case of plate with thickness $h=30 \mathrm{~mm}$ and $s=48 \mathrm{~mm} \times 48 \mathrm{~mm}$ for plate of thickness $h=10 \mathrm{~mm}$ is investigated for each defect.

Each defect is sensed with investigation probe positioned normally to the surface of inspected material. The lift-off parameter was set to $l_{\mathrm{o}}=1 \mathrm{~mm}$. The sensed data are acquired using the data acquisition card (DAQ) with the sampling frequency of $f_{\mathrm{s}}=15 \mathrm{kS} / \mathrm{sec}$ and the resolution of res $=16$ bits/channel. Using the LabVIEW environment is designed user interface for data manipulation,

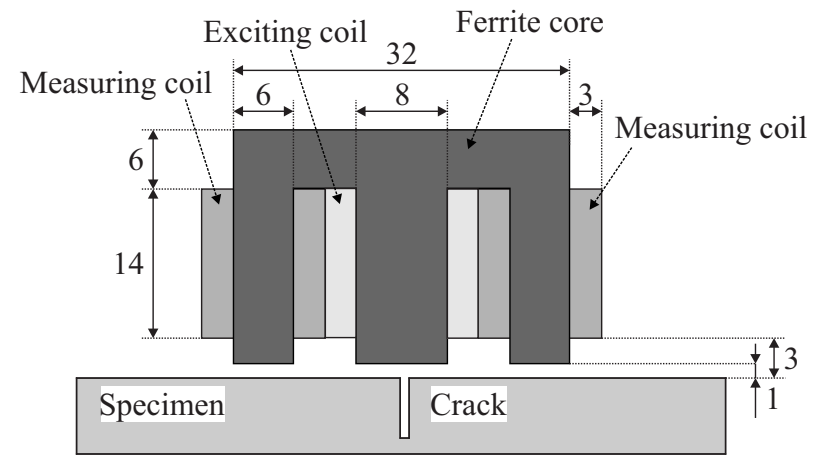

Fig. 4. PECT probe layout

controlling the stage and processing the data. Configuration of the experimental set-up is shown in Fig. 2.

\section{Testing equipment}

\section{ECT probe}

The probe used for ECT consists of two exciting coils connected in series and sensing element, Fig. 3. Probe is positioned normally to the surface of inspected material. In order to decrease coupling between the exciting and the sensing system exciting coils are connected in opposite magnetic orientation to each other.

The self-inductances of exciting coils are

$$
\begin{aligned}
& L_{1}=2.24 \mathrm{mH}, \\
& L_{2}=2.23 \mathrm{mH}
\end{aligned}
$$

and are driven by the harmonic current with an effective value of $I=0.6 \mathrm{~A}$ at frequency of $f_{1}=1 \mathrm{kHz}$.

\section{PECT probe}

The probe used for PECT, Fig. 4, consists of one excitation coil and two receiving coils that are wound on an E-shaped ferromagnetic core. The receiving coils are electrically connected and the output signal is measured as differential voltage of both coils.

Self-inductances of receiving coils are $L_{1}=4.5 \mathrm{mH}$, $L_{2}=4.6 \mathrm{mH}$ and self-inductance of the exciting coil is $L_{3}=2.35 \mathrm{mH}$. The exciting coil is driven by the pulsed voltage with a maximum value of $U_{\mathrm{m}}=8 \mathrm{~V}$ and a frequency of $f_{1}=1 \mathrm{kHz}$. Source voltage pulse with width $t_{\mathrm{d}}=0.1 \mathrm{~ms}$, rise time $t_{\mathrm{r}}=10 \mathrm{~ns}$ and fall time $t_{\mathrm{f}}=10 \mathrm{~ns}$ is used.

\section{ECT}

\section{Methods}

The created algorithm for both the length estimation uses the Wavelet transform,

$$
X_{W}(s, b)=\frac{1}{\sqrt{s}} \int_{-\infty}^{+\infty} x(t) \psi^{*}\left(\frac{t-b}{s}\right) \mathrm{d} t
$$


Table 2. The ECT and PECT obtained results for the plate with different thickness, near-side

\begin{tabular}{|c|c|c|c|c|c|c|c|c|c|c|c|c|}
\hline \multirow[b]{3}{*}{ Defect } & \multicolumn{6}{|c|}{ ECT } & \multicolumn{5}{|c|}{ PECT } & \multirow[b]{3}{*}{$d_{e}$} \\
\hline & \multicolumn{3}{|c|}{$h=30$} & \multicolumn{3}{|c|}{$h=10$} & \multicolumn{2}{|c|}{$h=30$} & \multicolumn{3}{|c|}{$h=10$} & \\
\hline & $l_{e}$ & $d_{c}$ & $d_{e}$ & $l_{e}$ & $d_{c}$ & $d_{e}$ & $l_{e}$ & $d_{c}$ & $d_{e}$ & $l_{e}$ & $d_{c}$ & \\
\hline 1 & 30 & 5 & 5.1 & 10 & 1 & 1.2 & 29.5 & 5 & 5.5 & 9.5 & 1 & 1 \\
\hline 2 & 30.1 & 10.3 & 10.2 & 10.5 & 3 & 3 & 30 & 10.3 & 10.5 & 10 & 3 & 3 \\
\hline 3 & 29.8 & 16.3 & 16.5 & 10.5 & 5 & 5.1 & 30.5 & 16.3 & 15 & 10.5 & 5 & 4.5 \\
\hline 4 & 30 & 20.5 & 20.2 & 11 & 7 & 7.5 & 31 & 20.5 & 20 & 11 & 7 & 7.5 \\
\hline 5 & 29.9 & 24.5 & 25.3 & 11.5 & 9 & 8.8 & 31 & 24.5 & 25.5 & 10.5 & 9 & 9.5 \\
\hline
\end{tabular}

Table 3. The ECT and PECT obtained results for the plate with different thickness, far-side

\begin{tabular}{|c|c|c|c|c|c|c|c|c|}
\hline \multirow[b]{3}{*}{ Defect } & \multicolumn{4}{|c|}{ ECT } & \multicolumn{4}{|c|}{ PECT } \\
\hline & \multicolumn{2}{|c|}{$h=30$} & \multicolumn{2}{|c|}{$h=10$} & \multicolumn{2}{|c|}{$h=30$} & \multicolumn{2}{|c|}{$h=10$} \\
\hline & $l_{e}$ & $d$ & $l_{e}$ & $d$ & $l_{e}$ & $d$ & $l_{e}$ & $d$ \\
\hline 1 & 29.5 & 5 & 10.5 & 1 & 32 & 5.5 & 10 & 1 \\
\hline 2 & - & 10.3 & 11.5 & 3 & 33.5 & 9.5 & 10.5 & 3 \\
\hline 3 & - & 16.3 & 13 & 5 & 36 & 13.7 & 11.5 & 5 \\
\hline 4 & - & 20.5 & - & 7 & - & 19.7 & 12.5 & 7 \\
\hline 5 & - & 24.5 & - & 9 & - & 25 & 14 & 9 \\
\hline
\end{tabular}

with Haar mother wavelet.

A convolution process was applied to individual signal matrices. Estimated defect parameters were stated as a sum of the individual scalegrams. The results based on the Wavelet transform showed that the length of the defects were estimated by use of integral sensor [3].

Here $x(t)$ is vector of measure data, $\psi(t)$ is mother wavelet, $s$ is scale and $b$ is shift of the computing window. The estimated depth of defect was determined by the neural network. The input matrix data was reduced by principle component analysis. For training function Bayesian regularization backpropagation (BRB) was used, which updates the weight and bias values according to Levenberg-Marquardt optimization. First it minimizes a combination of squared errors and weights, and then determines the correct combination. This process produces a network that generalizes well and it is called Bayesian regularization. Following formula for output of the neural networks had been used for solution of the inverse problem in the case of depth and implemented in Matlab, where $y$ is value of the output, $x$ is value of the input, $w$ is synaptic weight, $p$ is threshold and $f$ is activating function $[2,3]$

$$
y=f\left(\sum_{j=1}^{n} w_{j} x_{j}-p\right) .
$$

\section{PECT}

For PECT algorithm based on cross-correlation of measured data matrix with samples of known reference signals was applied. Cross-correlation is a mathematical method of estimating similarity of two data series as a function of their relative displacement. In presented case first data set is matrix of measured data and second is short reference signal acquired as a system response on pulsed excitation above defect of known parameters. Following formula for discrete cross-correlation had been used and implemented in Matlab [7]

$$
R_{x y}=\sum_{n=0}^{N-1} \mathbf{x}(n) \cdot \boldsymbol{y}(n-k)
$$

where $\boldsymbol{y}(n-k)$ is row vector of measured data matrix, $\mathbf{x}(n)$ is reference signal and $R_{x y}$ is output crosscorrelation product. Cross-correlation product exhibits distinctive peaks in the areas of high degree of similarity. Cross-correlation product peaks were then detected and compared with database of known reference signals in terms of area, amplitude and tailing factor to estimate the depth of the defect.

Cross-correlation product exhibits distinctive peaks in the areas of high degree of similarity. Cross-correlation product peaks were then detected and compared with database of known reference signals in terms of area, amplitude and tailing factor to estimate the depth of the defect.

\section{Computing time}

The computing time is very important factor for deciding which method will use for solution with the in- 


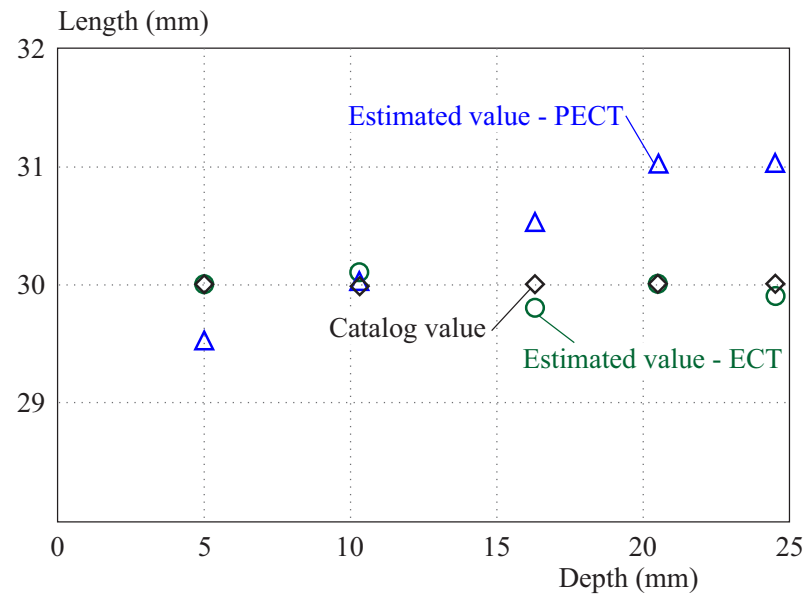

Fig. 5. Defect length for plate with $h=30 \mathrm{~mm}$, near-side

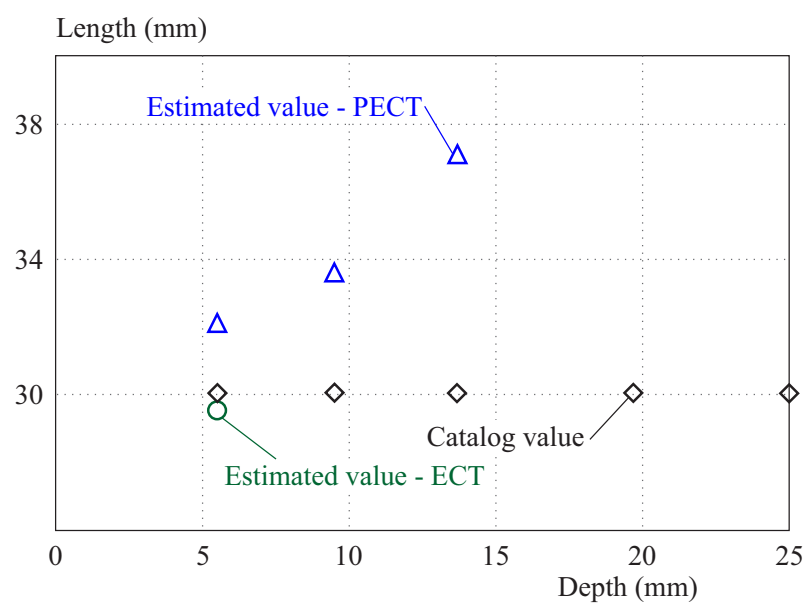

Fig. 7. Defect length for plate with $h=30 \mathrm{~mm}$, far-side

verse problem in the non-destructive testing. Conventional methods are time-consuming, therefore two procedures have been developed to determine the geometric parameters of the inspected defects. These ones are focused on reduction of the computing time in order to keep accuracy of the estimation. In the case of the ECT method, the average computing time is equal $t_{c}=16 \mathrm{~s}$. On the other side the computing time for PECT method is $t_{c}=47 \mathrm{~s}$.

\section{Results and discussion}

Near-side measurement

The results for ECT near-side measurement of the defects are represented in Tab. 2 (the plate of thickness $h=30 \mathrm{~mm}$ and $h=10 \mathrm{~mm}$ ). The symbols $l_{c}, l_{e}, d_{c}$ and $d_{e}$ mean catalog value of the defects length, estimated length, catalog value of the defect depth and estimated depth, respectively.

Estimated length of surface defect as a function of its depth is shown in Figs. 5 and 6. For ECT maximum

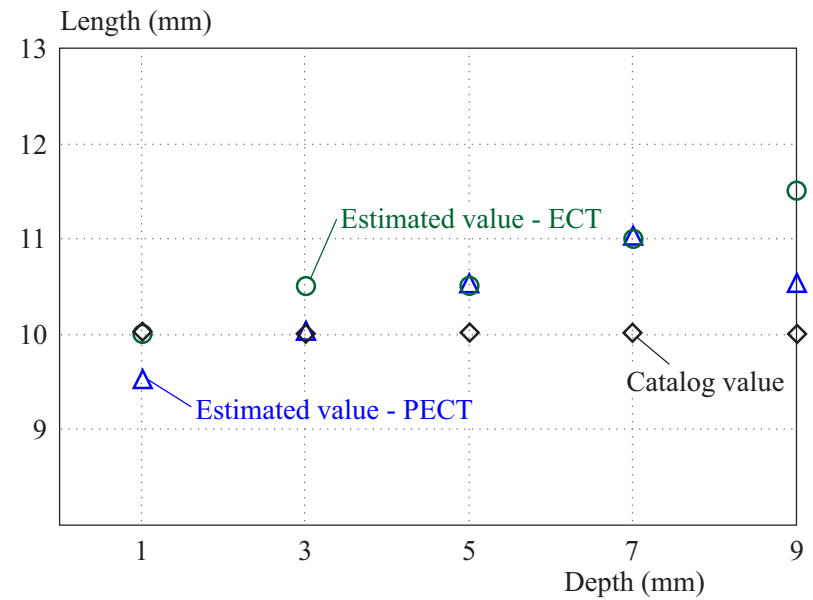

Fig. 6. Defect length for plate with $h=10 \mathrm{~mm}$, near-side

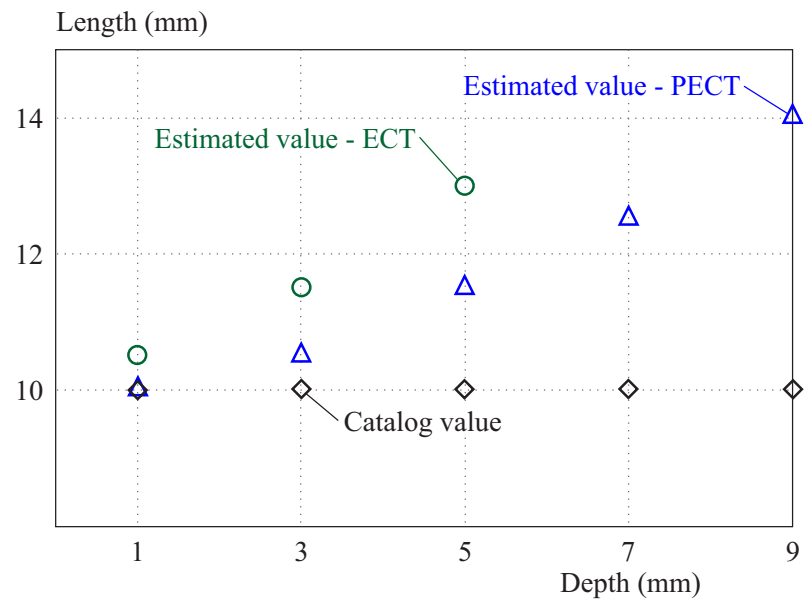

Fig. 8. Defect length for plate with $h=10 \mathrm{~mm}$, far-side

relative error of estimation is $0.7 \%$ for plate with $h=$ $30 \mathrm{~mm}$ and $15 \%$ for plate with $h=10 \mathrm{~mm}$. For PECT maximum relative error of estimation is $3 \%$ for plate with $h=30 \mathrm{~mm}$ and $10 \%$ for plate with $h=10 \mathrm{~mm}$. For both methods these errors occur during the investigation of the deepest defects of the plate.

Far-side measurement

The results for ECT far-side measurement of the defects are represented in Tab. 3. (the plate with thickness $h=30 \mathrm{~mm}$ ) and the plate with thickness $h=10 \mathrm{~mm}$ ). The created algorithm was used for solution of the inverse problem in this case. The subsurface defects was measured same way as surface defects. The symbol $d$ means the depth at which the defect is located.

The skin effect is the limit which influence the use of the eddy current testing methods. Therefore, the defects which are deep-lying $(5 \mathrm{~mm}$ and more in the subsurface for ECT and $13.7 \mathrm{~mm}$ for PECT) do not influences the flow pattern of the eddy currents enough to be detectable by the eddy current methods. 
Estimated length of subsurface defect as a function of its depth is shown in Figs. 7 and 8. For both methods relative error of estimation of subsurface defect dimensions substantially increases with its distance from surface up to $2 \%$ for plate with thickness $h=30 \mathrm{~mm}$ and $30 \%$ for the plate with thickness $h=10 \mathrm{~mm}$, on the other side, relative error of PECT estimation is up to $24 \%$ for plate with thickness $h=30 \mathrm{~mm}$ and $40 \%$ for plate with thickness $h=10 \mathrm{~mm}$.

\section{Conclusions}

Presented study compares two approaches to nondestructive testing with use of eddy currents as an evaluation tool for surface and subsurface defects. Both methods are affected by skin effect, which limits their application and depth of detectable defects.

For PECT measured limit of detectable subsurface defect is $13.7 \mathrm{~mm}$. This depth is enabled by wide range of harmonics in pulsed rectangular signal and restoration of system response by implementation of cross-correlation with the ideal reference signal. This operation allow us to evaluate even highly distorted output signals. Disadvantage of PECT method lies in necessity of accurately capture of output signal shape, which is necessary for cross-correlation. That means higher sampling rate and longer computing time. This method also show lower estimation error for subsurface defects for plate with width $h=10 \mathrm{~mm}$.

On the other side, the ECT detectable limit is $d=$ $5 \mathrm{~mm}$ which means distance located the defect from surface. These limits are possible decrease in the case if we used lower excitation frequency, but main disadvantage of the lower frequency is lower resolution and decreasing accuracy. Based on the obtained results, it can be concluded that the ECT method and created algorithm (Wavelet transform and Neural network) achieved a lower relative error for estimation of the geometric parameters of surface defects for both samples than PECT method and cross-correlation analysis. However, this method is more limited by skin effect than PECT method.

\section{REFERENCES}

[1] S. J. Norton and J. R. Bowler, "Eddy Current Interaction with an Ideal Crack, II: The Inverse Problem", Journal of Applied Physics vol. 75, 1994, pp. 8138.

[2] A. Pirani, M. Ricci, A. Tamburrino and S. Ventre, "3D Reconstruction of Flaws in Metallic Materials by Eddy Current Inspections", Electromagnetic Nondestructive Evaluation vol. XI, IOS Press, 2008, Studies in Applied Electromagnetics and Mechanics 31, .

[3] D. Gombarska, B. Babusiak and M. Gala, "Signal Processing in the Medicine", 1st edition. Žilina, EDIS - Publishing ŽU, 2013.

[4] M. Smetana, V. Chudacik, R. Konar and M. Mician, "Austenitic Biomaterial Cracks Evaluation by Advanced Nondestructive Techniques", Advances in Electrical and Electronic Engineering 2017.

[5] H. Yunze, L. Feilu and P. Mengchun, "Defect Characterization Based on Pulsed Eddy Current Imaging Technique", Sensors and Actuators A, Physical, vol. 164, 2010, pp. 1-7.

[6] S. Laia, D. Y. Chena, H. Chena and Y. W. Fub, "Pulsed Eddy Current Testing of Inner Wall Flaws in Pipe under Insulation", Procedia Engineering, vol. 30, 2015, pp. 1658-1664.

[7] M. M. Daniel and A. C. Singer, "Computer Explorations in Signals and Systems Using MATLAB", Upper Saddle River, NJ: Prentice Hall, 2002.

Received 26 September 2018 